\title{
Philosophiques
}

\section{La vision unificatrice de Grothendieck : au-delà de l'unité (méthodologique ?) des mathématiques de Lautman}

\section{Mathieu Bélanger}

Volume 37, numéro 1, printemps 2010

Albert Lautman, philosophe des mathématiques

URI : https://id.erudit.org/iderudit/039718ar

DOI : https://doi.org/10.7202/039718ar

Aller au sommaire du numéro

Éditeur(s)

Société de philosophie du Québec

ISSN

0316-2923 (imprimé)

1492-1391 (numérique)

Découvrir la revue

Citer cet article

Bélanger, M. (2010). La vision unificatrice de Grothendieck : au-delà de l'unité (méthodologique ?) des mathématiques de Lautman. Philosophiques, 37(1),

169-187. https://doi.org/10.7202/039718ar
Résumé de l'article

Dans sa thèse complémentaire intitulée « Essai sur l'unité des sciences mathématiques dans leur développement actuel » Albert Lautman analysa la question de l'unité des mathématiques en considérant différentes paires antithétiques de concepts mathématiques, notamment le continu et le discret. Dans le cadre de sa refonte de la géométrie algébrique abstraite, le mathématicien français Alexandre Grothendieck considéra également l'opposition traditionnelle du continu et du discret selon un cadre conceptuel fort similaire à celui de Lautman. En comparaison, l'introduction du concept de topos lui permit de donner une réponse strictement mathématique et parfaitement claire à cette question. 


\title{
La vision unificatrice de Grothendieck: au-delà de l'unité (méthodologique?) des mathématiques de Lautman
}

\author{
MATHIEU BÉLANGER \\ mathieu.belanger@umontreal.ca \\ Université de Montréal
}

\begin{abstract}
RÉSUMÉ. - Dans sa thèse complémentaire intitulée «Essai sur l'unité des sciences mathématiques dans leur développement actuel» Albert Lautman analysa la question de l'unité des mathématiques en considérant différentes paires antithétiques de concepts mathématiques, notamment le continu et le discret. Dans le cadre de sa refonte de la géométrie algébrique abstraite, le mathématicien français Alexandre Grothendieck considéra également l'opposition traditionnelle du continu et du discret selon un cadre conceptuel fort similaire à celui de Lautman. En comparaison, l'introduction du concept de topos lui permit de donner une réponse strictement mathématique et parfaitement claire à cette question.
\end{abstract}

\begin{abstract}
Albert Lautman's complementary thesis, entitled "Essai sur l'unité des sciences mathématiques dans leur développement actuel", analysed the unity of mathematics through various antithetical pairs of mathematical concepts such as the continuous and the discrete. As part of his renewal of abstract algebraic geometry, French mathematician Alexandre Grothendieck also considered the traditional opposition between the continuous and the discrete and adopted a conceptual framework very similar to that of Lautman. However, the topos concept allowed Grothendieck to come up with a purely mathematical and, in comparison, much clearer solution.
\end{abstract}

En termes philosophiques, la question de l'unité des mathématiques peut être abordée de différents points de vue. Par exemple, la théorie des ensembles met de l'avant une unité ontologique dans la mesure où elle considère tous les objets mathématiques comme étant des ensembles. Pour sa part, la théorie des catégories suggère une unité épistémologique. En effet, en théorie des catégories, la connaissance d'un objet mathématique s'acquiert par l'entremise des morphismes qui le relient à d'autres objets ${ }^{1}$.

La thèse complémentaire d'Albert Lautman, complétée en 1937 et intitulée «Essai sur l'unité des sciences mathématiques dans leur développement actuel », privilégie également un point de vue philosophique sur la question de l'unité des mathématiques. À cet égard, la contribution de Lautman s'avère fort singulière et originale en ce qu'il avance une unité métaphysique. La proposition de Lautman est d'autant plus singulière que la problématique y est

1. Ces exemples sont dus à Jean-Pierre Marquis. 
campée à l'aide de paires de concepts antithétiques qui sont quant à eux résolument mathématiques.

Pour cette raison, le présent article s'affaire à mettre en parallèle la position métaphysique de Lautman sur l'unité des mathématiques avec celle du mathématicien français Alexandre Grothendieck. En effet, Grothendieck envisagea la question de l'unité des mathématiques en des termes mathématiques similaires à ceux de Lautman. En contrepartie, le concept de topos lui permit de résoudre l'opposition antithétique fondamentale à l'aide des mathématiques elles-mêmes.

\section{L'unité des mathématiques chez Lautman}

Lautman envisage le problème de l'unité des mathématiques sous la forme d'un commentaire de la préface du livre Gruppentheorie und Quantenmechanik de Hermann Weyl. S'inspirant de Weyl, Lautman développe une opposition entre deux pôles de concepts dont la réconciliation dévoilerait l'unité à l'œuvre en mathématiques.

\subsection{Le problème de l'unité des mathématiques}

L'«Essai sur l'unité des sciences mathématiques dans leur développement actuel» s'ouvre sur une longue citation de Weyl où sont distinguées deux tendances dans le développement historique des mathématiques ${ }^{2}$. La première, héritée des traditions indienne et arabe, se caractérise par un système de nombres universel. Les mathématiques dites "classiques", c'est-à-dire l'analyse tel qu'il se développa jusqu'à la théorie des fonctions de variable complexe, en sont le fruit. La seconde, que Weyl associe aux Grecs, affirme que tout domaine donne lieu à un système de nombres lui étant propre. La coexistence de plusieurs systèmes de nombres, chacun étant associé à des domaines différents, devient alors possible. L'algèbre abstraite, et plus particulièrement la théorie des groupes, est l'archétype de ce courant qu'il convient d'appeler mathématiques «modernes».

Lautman voit dans cette distinction deux courants au sein des mathématiques contemporaines.

[Weyl] affirme de la façon la plus nette l'existence d'une division essentielle dans les mathématiques contemporaines; il faudrait en effet distinguer l'une de l'autre la mathématique «classique» qui, partant de la notion de nombre entier aboutit à l'analyse, et la mathématique «moderne» qui s'opposant à la mathématique des nombres, affirme au contraire le primat de la notion de domaine par rapport aux nombres attachés à ce domaine. [1977b, p. 155-156]

Lautman oppose deux pôles qu'il fait correspondre aux mathématiques «classiques» et aux mathématiques «modernes». La caractérisation que donne Lautman de ces pôles s'avère malheureusement assez confuse

2. Voir Lautman [1977b , p. 155-156]. 
dans la mesure où elle englobe des considérations historiques, méthodologiques, disciplinaires, etc. et se trouve du coup à jouer sur plusieurs niveaux simultanément. Cette opposition entre deux pôles est à un moment ou l'autre caractérisée par les paires suivantes ${ }^{3}$ :

\begin{tabular}{l|l} 
mathématique classique & mathématique moderne \\
\hline $\begin{array}{l}\text { primat du nombre } \\
\text { mathématiques du XIX }{ }^{\mathrm{e}} \text { siècle } \\
\text { point de vue local }\end{array}$ & $\begin{array}{l}\text { primat du domaine } \\
\text { mathématiques du } \mathrm{XX}^{\mathrm{e}} \text { siècle } \\
\text { approche constructiviste } \\
\text { arithmétique et analyse } \\
\text { nature numérique des objets }\end{array}$ \\
$\begin{array}{l}\text { approche axiomatique } \\
\text { arithmétique et algèbre moderne }\end{array}$ & nature abstraite des objets
\end{tabular}

Nonobstant cette confusion et contrairement à ce que la citation cidessus pourrait laisser croire, l'opposition ne semble pas être historique, c'est-à-dire que les pôles des mathématiques "classiques » et "modernes " ne peuvent être assimilés respectivement à celles du XIX ${ }^{e}$ siècle et du $\mathrm{XX}^{\mathrm{e}}$ siècle. En effet, la division se situe au sein des mathématiques contemporaines. D'autre part, le cas de la topologie fait également dire à Lautman que cette opposition ne se réduit pas à un renversement en faveur du primat du concept de domaine sur celui de nombre — tout important cet aspect soit-il. Bien que les propriétés des fonctions dépendent de leurs domaines de définition, Lautman ne manque pas de souligner que «[1]a distinction que Weyl établit entre l'analyse classique et la mathématique géométrique moderne ne saurait donc tendre à opposer les unes aux autres les méthodes de la théorie des fonctions et celles de la topologie; ce sont les mêmes (...)» [1977b, p. 158]

Le propos de la thèse suggère plutôt que, aux yeux de Lautman, la distinction fondamentale réside entre l'analyse classique et l'algèbre abstraite, cette dernière étant comprise selon la conception popularisée par van der Warden dans son célèbre livre Moderne Algebra.

À cet égard, Lautman met en évidence quatre aspects qui caractérisent l'algèbre abstraite ${ }^{4}$ :

1) Priorité de la structure globale d'un domaine par rapport aux nombres attachés à ce domaine;

2) Priorité de la notion de domaine sur celle de nombre;

3) Possibilité de multiplications non commutatives;

4) Caractère fini et discontinu des structures algébriques.

3. Cf. Lautman [1977b , p. 159].

4. Pour une description plus détaillée de chacun de ces aspects, voir Lautman [1977b, p. 158-160]. 
Lautman trace ainsi une distinction entre l'analyse classique et l'algèbre abstraite et, du coup, donne également une caractérisation de l'opposition qui l'intéresse, à savoir celle entre le pôle des mathématiques "classiques" et celui des mathématiques "modernes».

Le problème de l'unité des mathématiques se résume alors à l'interprétation de cette opposition entre l'analyse classique et l'algèbre abstraite. Lautman envisage deux possibilités:

Les méthodes et les conceptions fondamentales de l'algèbre moderne étant ainsi distinguées de celles de l'analyse, on peut chercher à interpréter le sens de cette dualité qui apparaît profondément installée au sein des mathématiques contemporaines. Est-ce une dualité essentielle entre théories irréductibles les uns [sic] aux autres ou bien n'est-ce qu'une dualité de méthodes susceptibles d'être un jour rapprochées? [1977b, p. 160]

Se réclamant de Hilbert, la position de Lautman est claire: il n'y a pas de dualité essentielle au sein des mathématiques, mais bien une unité.

\subsection{Une unité méthodologique?}

Lautman affirme que, dans une perspective mathématique, l'unité qu'il envisage est méthodologique, c'est-à-dire que l'unité se réalise au niveau des méthodes employées par les mathématiciens. Selon Lautman, les mathématiques contemporaines dissipent l'opposition entre l'analyse et l'algèbre abstraite par le recours à des méthodes typiques de cette dernière pour la résolution de problèmes d'analyse.

Nous nous proposons dans les pages qui vont suivre de montrer comment la mathématique moderne est engagée dans la voie de cette unification de l'algèbre et de l'analyse et ceci par la pénétration de plus en plus poussée des méthodes structurales et finitistes de l'algèbre dans le domaine de l'analyse et du continu. En somme le conflit des méthodes entre l'algèbre et l'analyse se dissiperait au profit de l'algèbre; la distinction des deux mathématiques de Weyl semble ainsi ne correspondre qu'aux conditions historiques du développement des mathématiques et laisser intacte l'unité des mathématiques et l'unité de l'intelligence. [Lautman 1977b, p. 160]

La thèse complémentaire prend alors la forme d'un long argument visant à exemplifier cette infiltration des méthodes algébriques en analyse. Chacun des quatre chapitres aborde un des aspects caractérisant l'algèbre abstraite de façon à montrer que celui-ci est à l'œuvre dans les recherches contemporaines d'analyse. Voici la présentation que donne Lautman de son argument :

Nous nous proposons dans les quatre chapitres de cet ouvrage de montrer comment on peut retrouver dans les théories modernes de l'analyse les aspects qui nous ont paru caractériser l'algèbre moderne: L'idée [sic] algébrique de dépendance d'une grandeur par rapport au domaine auquel elle appartient nous a amené à étudier l'influence de la structure «dimensionnelle» d'un 
ensemble sur le mode de décomposition individuelle de ses éléments et à envisager l'importance des décompositions dimensionnelles en théorie de fonctions (chapitre Ir) La priorité de la topologie de certains domaines sur les nombres attachés à ces domaines se retrouve dans le rôle des métriques non euclidiennes de la théorie des fonctions analytiques (chapitre II); nous étudions ensuite (chapitre III) l'algèbre non commutative qui intervient dans la théorie de l'équivalence des équations différentielles. Enfin nous montrons comment la considération de structures algébriques finies et discontinues peut servir à déterminer l'existence de fonctions de variable continue (chapitre IV). [1977b, p. 160]

Dans les faits, chacun des quatre chapitres aborde effectivement un des quatre aspects l'algèbre moderne identifiés par Lautman. En contrepartie, l'identification de ces aspects dans les exemples mathématiques présentés pose parfois problème. Dans les trois premiers chapitres, les différents exemples présentés insistent d'abord et avant tout sur la réduction de problèmes d'analyse à des problèmes d'algèbre. Par le fait même, les considérations annoncées à la fin de l'introduction - voir la citation précédente - sont reléguées à l'arrière-plan au profit de la présentation de moult théories ou exemples mathématiques.

Par exemple, l'exemple central du premier chapitre est la théorie des fonctions linéaires intégrales telle que développée par Hilbert.

Soit l'équation linéaire intégrale

$$
f(s)=\varphi(s)-\lambda \int_{0}^{1} k(s, t) \varphi(t) d t
$$

où $\varphi$ est la fonction recherchée. La résolution de cette équation peut être ramenée à la résolution d'un système d'équations algébriques. Pour ce, l'approche de Hilbert consiste à considérer un système de fonctions continues orthonormales $\omega_{p}(s)(0 \leq s \leq 1)$ qui satisfassent la condition suivante relativement à une fonction continue $u(s)$ :

$$
\int_{0}^{1} u(s)^{2} d s=\left[\int_{0}^{1} u(s) \omega_{1}(s) d s\right]^{2}+\left[\int_{0}^{1} u(s) \omega_{2}(s) d s\right]^{2}+\ldots
$$

Le système des fonctions $\omega_{p}(s)$ forme une base pour l'espace des fonctions $u(s)$. Sont alors construites les expressions

$$
\begin{array}{ll}
x_{p}=\int_{0}^{1} \varphi(s) \omega_{p}(s) d s & f_{p}=\int_{0}^{1} f(s) \omega_{p}(s) d s \\
k_{q}=\int_{0}^{1} k(s, t) \omega_{q}(t) d t & k_{p q}=\int_{0}^{1} \int_{0}^{1} k(s, t) \omega_{p}(s) \omega_{q}(t) d s d t
\end{array}
$$

telles que les séries $\sum x_{p}^{2}, \sum f_{p}^{2}, \sum k_{q}(s)^{2}$ et $\sum k_{p q}(s)^{2}$ convergent. 


\section{4 • Philosophiques / Printemps 2010}

Sous ces conditions, le système d'une infinité équations linéaires algébriques à une infinité de variables suivant se déduit de l'équation intégrale de départ:

$$
\begin{gathered}
\left(1-\lambda k_{11}\right) x_{1}-\lambda k_{12} x_{2}-\cdots-\lambda k_{1 n} x_{n} \cdots=f_{1} \\
-\lambda k_{21} x_{1}+\left(1-\lambda k_{22}\right) x_{2}-\cdots-\lambda k_{2 n} x_{n} \cdots=f_{2} \\
\vdots \\
-\lambda k_{m 1} x_{1}-\lambda k_{m 2} x_{2}-\cdots+\left(1-\lambda k_{m n}\right) x_{n} \cdots=f_{m}
\end{gathered}
$$

Ainsi, le problème d'analyse originel — trouver la solution d'une équation intégrale - se réduit à un problème algébrique - trouver la solution d'un système d'équations algébriques.

En effet, toute solution du système d'équations algébriques ci-dessus permet d'obtenir une solution de l'équation intégrale. Soit $x_{1}, x_{2}, x_{3}, \ldots$ une solution de ce système. Il suffit de former la série uniformément convergente suivante pour obtenir une solution de l'équation intégrale:

$$
\varphi(s)=f(s)+\lambda \sum_{q} k_{q}(s) x_{q}
$$

Or, cette solution dépend de ce que Lautman appelle la structure dimensionnelle de l'espace. En effet, la fonction $\varphi$ admet un développement en série de Fourier:

$$
\varphi(s)=\sum_{p=1}^{\infty} x_{p} \omega_{p}(s)
$$

où les coefficients sont donnés par

$$
x_{p}=\int_{0}^{1} \varphi(s) \omega_{p}(s) d s
$$

Le point important est que le développement en série de Fourier se fait relativement à la base de l'espace des fonctions $u(s)$, c'est-à-dire au système de fonctions orthonormales $\omega_{p}$. Ceci signifie que la structure de l'espace coordonne le processus de réduction du problème d'analyse à un problème d'algèbre.

Le quatrième et dernier chapitre emprunte une voie différente des précédents. Là où les trois premiers chapitres montraient comment des problèmes d'analyse se réduisaient à des situations algébriques, le quatrième se tourne vers la question d'une théorie des rapports du continu et du discret. La perspective de Lautman est la suivante: «(...) étudier, au sein de la théorie analytique des nombres elle-même, le mécanisme des liaisons qui s'y affirment entre le continu et le discontinu.» [1977b, p. 188] 
Lautman présente deux situations mathématiques à cet égard. La première est la fonction zêta sur un corps $K$, c'est-à-dire la fonction

$$
\zeta_{K}(s)=\sum \frac{1}{[N(\mathcal{A})]^{s}}
$$

définie sur tous les idéaux entiers $\mathcal{A}$ de $K$ où $s$ est une variable complexe telle que $s>1$ et $N(\mathcal{A})$ désigne la norme d'un idéal $\mathcal{A}$.

Cette fonction peut également s'écrire sous la forme d'un produit

$$
\zeta_{K}(s)=\prod \frac{1}{1-\frac{1}{[N(\mathcal{J})]^{s}}}
$$

portant sur tous les idéaux premiers de $K$.

Lautman rappelle d'abord que la fonction zêta permet d'obtenir différentes propriétés des idéaux du corps $K$, notamment qu'il existe une infinité d'idéaux premiers dans toute classe d'idéaux de $K$.

Ceci étant posé, Lautman affirme que «l'utilisation arithmétique sur le corps $K$ de la fonction $\zeta_{\mathrm{K}}(s)$ n'est possible que parce que la détermination de cette fonction implique déjà la connaissance de certaines propriétés structurales et discontinues du corps de base.» [1977b, p. 191]

Par exemple, l'existence dans toute classe d'idéaux de $K$ d'une infinité d'idéaux premiers dépend de la formule

$$
\lim _{s=1}(s-1) \zeta_{K}(s)=h \kappa
$$

où $h$ est le nombre de classes du corps $K$ et $\kappa$ un invariant de $K$. Ainsi, la fonction $\zeta$ dépend de la décomposition du corps $K$ en ses classes d'idéaux.

Ceci signifie que la fonction $\zeta_{\mathrm{K}}(s)$ existe en vertu de la structure algébrique du corps $K$ et qu'elle permet à son tour d'étudier $K$. Comme le dit Lautman:

En somme on obtient le schéma suivant: la décomposition en classes du corps $K$ permet de conclure à l'existence de la fonction $\zeta_{\mathrm{K}}(s)$ dont la connaissance peut, par choc en retour sur le corps de base, servir à une étude plus approfondie de ce corps. [1977b, p. 191]

La seconde situation présente la dépendance des fonctions automorphes relativement au groupe modulaire. Un schéma similaire y est met en évidence.

À la lumière de ces considérations, le quatrième chapitre semble marquer un changement de point de vue sur l'unité des mathématiques, changement auquel il importe de s'attarder.

1.3 L'unité des mathématiques à travers la dualité du continu et du discret Dans l'introduction et les trois premiers chapitres de l'«Essai sur l'unité des sciences mathématiques dans leur développement actuel », Lautman tente de 
dissiper l'opposition entre l'analyse classique et l'algèbre abstraite. L'unité des mathématiques s'incarnerait ainsi dans la réconciliation de l'analyse et de l'algèbre. Toutefois, les considérations développées dans le quatrième chapitre laissent présager que cette opposition dépendrait d'une autre opposition encore plus fondamentale, c'est-à-dire la dualité du continu et du discret.

Lautman écrit au tout début du quatrième chapitre que les pôles des mathématiques «classiques » et «modernes » correspondent respectivement au continu et au discret.

Nous avons vu dans l'Introduction de cet ouvrage comment la distinction des deux mathématiques de Weyl pouvait être interprétée en termes d'une opposition entre la mathématique du fini et celle de l'infini ou plus exactement du dénombrable et du continu. [1977b, p. 187]

Ceci constitue certainement un changement de point de vue puisque, malgré l'affirmation de Lautman à cet effet, la dualité du continu et du discret n'était certainement pas présente explicitement dans l'introduction.

La conclusion de l'«Essai sur l'unité des sciences mathématiques dans leur développement actuel » confirme ce changement de point de vue; l'unité des mathématiques doit être considérée à travers la dualité du continu et du discret:

Il est donc légitime d'opposer la mathématique du $\mathrm{Xx}^{\mathrm{e}}$ siècle à celle du $\mathrm{XIX}^{\mathrm{e}}$ siècle, mais elles s'opposent comme la physique du continu et du discontinu. Il existe ainsi une certaine analogie entre la physique contemporaine et la mathématique contemporaine, en ce sens qu'elles offrent l'une et l'autre le spectacle de faits susceptibles d'être à la fois étudiés par le calcul du continu et par le calcul du discontinu, mais alors que cette dualité de méthodes relatives aux mêmes faits est la source des principales difficultés de la physique contemporaines, elle est au contraire le témoignage de l'unité profonde des mathématiques. [Lautman 1977b, p. 195]

Deux remarques s'imposent à propos de cette citation. Premièrement, elle confirme que la tension fondamentale en est bel et bien une entre le continu et le discret. Malheureusement, son interprétation est compliquée — encore une fois - par le flottement terminologique de Lautman. Tout au long du quatrième chapitre et de la conclusion, les paires de termes qu'oppose Lautman varient fréquemment au point que chacune des suivantes soit utilisée au moins une fois:

\begin{tabular}{l|l} 
pôle du continu & pôle du discret \\
\hline continu & discontinu \\
infini & fini \\
continu & dénombrable \\
analyse & arithmétique \\
analyse & algèbre \\
mathématiques du xix & mathématiques du $\mathrm{Xx}^{\mathrm{e}}$ siècle
\end{tabular}


Ce flottement terminologique induit un flou conceptuel. En effet, le fini, le dénombrable et le discontinu sont assurément des concepts mathématiques qui ne peuvent être assimilés l'un à l'autre. Dans le même ordre d'idées, il serait simpliste de réduire la dualité du continu et du discret à celle de l'infini et du fini. Il s'agit de deux problématiques distinctes. Lautman amalgame donc des concepts forts différents. Ceci est d'autant plus surprenant que les nombreux exemples présentés dans «Essai sur l'unité des sciences mathématiques dans leur développement actuel », mais aussi dans les autres écrits de Lautman, traduisent une vaste culture mathématique et qu'il était sans aucun doute parfaitement conscient des différences fondamentales entre ces concepts.

Deuxièmement, l'analogie avec la physique insiste sur le caractère méthodologique de l'unité des mathématiques. Selon Lautman, la réalité physique peut être traitée du point de vue du continu ou encore du point de vue du discret. Selon le cas, elle est appréhendée respectivement par le biais d'outils mathématiques continus, c'est-à-dire en utilisant des équations différentielles, ou d'outils mathématiques discrets, c'est-à-dire la théorie des groupes, le calcul des matrices, les statistiques quantiques, etc. Nonobstant le point de vue et donc les méthodes privilégiés, il s'agit toujours de la même réalité physique qui est étudiée.

Par ricochet, les rapports du continu et du discret en physique suggèrent qu'une même réalité mathématique — au sens de l'objet d'étude du mathématicien - puisse être appréhendée de différentes façons. Étrangement, Lautman ne fait pas cette déduction dans la conclusion de sa thèse complémentaire. Il clôt cependant le premier chapitre avec une remarque à cet effet:

Les exemples que nous avons donnés nous permettent ainsi de comprendre que s'il y a des modes de pensée différents en mathématiques, il est peu vraisemblable qu'à ces différences de méthodes correspondent des différences de domaines. (...) Les mêmes êtres sont étudiables [sic] [de] deux façons et c'est cette rencontre des méthodes qui fait l'unité profonde des mathématiques. [1977b, p. 172]

Il y a ici un glissement: Lautman associe mode de pensée et méthode. Pourtant, des méthodes sont certainement différentes de modes de pensée. Les premières interviennent dans la manipulation d'objets mathématiques. Certaines peuvent prendre la forme de techniques standardisées, voire d'algorithmes, comme le calcul du déterminant d'une matrice $n \times n$, la construction d'une métrique à partir de la topologie d'un espace, la résolution d'une équation différentielle de deuxième ordre, etc. D'autres peuvent avoir une portée beaucoup plus vaste, notamment les méthodes de la théorie des catégories. La caractérisation d'un mode de pensée serait beaucoup plus délicate, mais, indépendamment de la réponse qui serait apportée à cette question, elle rattacherait probablement un mode de pensée à une activité intellectuelle. 
Ce glissement est révélateur dans la mesure où il indique que, selon Lautman et contrairement à ce que l'introduction et les quatre chapitres de la thèse complémentaire laissent croire, l'unité des mathématiques se réalise au niveau de la pensée.

Les dernières phrases de la thèse complémentaire vont dans cette direction en réintroduisant la problématique des schémas logiques au cœur de l' 'Essai sur les notions de structure et d'existence en mathématique » — sa thèse principale ${ }^{5}$. Selon Lautman, le continu et le discret s'inscrivent dans une relation dialectique qui balise le développement des mathématiques.

En s'attachant ainsi chaque fois, non pas à la quantité des éléments, mais à l'existence ou à l'armature des ensembles que l'on compare, on découvre ainsi entre le fini et l'infini des analogies de structures et des adaptations réciproques, d'où il résulte que l'unité des mathématiques est essentiellement celle des schémas logiques qui président à l'organisation de leurs édifices. Notre Essai sur les notions de structure et d'existence en mathématiques [sic] tend en effet à montrer qu'il est possible de retrouver au sein des théories mathématiques, des Idées logiques incarnées dans le mouvement même de ces théories. Les analogies de structures et les adaptations d'existences que nous avons essayé de décrire ici entre l'analyse et l'algèbre n'ont d'autre but que de contribuer à mettre en lumière l'existence au sein des mathématiques de schémas logiques, qui ne sont connaissables qu'à travers les mathématiques elles-mêmes, et en assurent à la fois l'unité intellectuelle et l'intérêt spirituel. [1977b, p. 198]

Chez Lautman, le problème de l'unité des mathématiques trouve donc sa solution, non pas dans les mathématiques elles-mêmes, mais au niveau de la pensée. Dans une perspective mathématique, la conception de l'unité des mathématiques présentée dans la thèse complémentaire, mais plus particulièrement la solution métaphysique au problème posé en termes mathématiques dans l'introduction, ne peut que laisser perplexe.

Cet aspect de l' «Essai sur l'unité des sciences mathématiques dans leur développement actuel» semble aussi avoir laissé perplexe Bernays comme l'exprime la conclusion du compte-rendu qu'il en publia en 1940:

In general, it might be thought that for a thorough inquiry into the relations of analysis to algebra and of the continuous to the discontinuous it would be more advantageous to go back to the primitive elements than to consider the theories which Lautman here takes as examples. But perhaps here the consideration of these theories is the main purpose and the philosophical theme is intended merely as a framework. [1940, p. 22]

L'idée d'une réconciliation du continu et du discret est également un des principaux thèmes de l'œuvre mathématique de Grothendieck. Cette réconciliation prend toutefois une forme totalement différente de celle avancée par Lautman dans son «Essai sur l'unité des sciences mathématiques dans leur développement actuel».

5. Voir Lautman [1977a]. 


\section{La vision unificatrice de Grothendieck}

Dans Récoltes et semailles, Grothendieck envisage les mathématiques selon un cadre conceptuel qui rappelle celui de Lautman. Toutefois, la question du rapport entre le discret et le continu y trouve une solution strictement mathématique. La structure des mathématiques elles-mêmes permet de rendre compte de l'unité du discret et du continu.

\subsection{Une vision des mathématiques}

Selon Grothendieck, trois aspects de la réalité mathématique sont traditionnellement distingués:

1) le nombre ou l'aspect arithmétique;

2) la grandeur ou l'aspect analytique, aussi appelé aspect métrique;

3) la forme ou l'aspect géométrique.

Il ajoute que, loin d'être mutuellement exclusifs, ces aspects se retrouvent dans la majorité des situations mathématiques et y sont en interaction. Ces aspects peuvent donc être compris comme autant de facettes de la réalité mathématique. En extrapolant, une situation donnée n'est pas intrinsèquement arithmétique - par exemple — mais est plutôt qualifiée d'arithmétique parce qu'envisagée sous cet aspect. Au nombre, à la grandeur et à la forme correspondent alors des points de vue par l'entremise desquels peut être appréhendée la réalité mathématique.

À l'exception des travaux d'analyse fonctionnel de ses débuts, Grothendieck considère que ses recherches logèrent à l'enseigne de la forme, c'est-à-dire qu'elles portèrent sur l'aspect géométrique des mathématiques. Plus particulièrement, Grothendieck s'intéressa à la forme telle qu'elle s'incarne dans les structures.

C'est dire que s'il y a une chose en mathématique qui (depuis toujours sans doute) me fascine plus que toute autre, ce n'est ni «le nombre», ni «la grandeur ", mais toujours la forme. Et parmi les mille-et-un visages que choisit la forme pour se révéler à nous, celui qui m'a fasciné plus que tout autre et continue à me fasciner, c'est la structure cachée dans les choses mathématiques. [Grothendieck 1985, p. P27] ${ }^{6}$

Ainsi, Grothendieck voit son travail mathématique comme ayant été voué au dévoilement des structures qui animent les choses mathématiques. À ses yeux, le mathématicien ne construit ni n'invente des structures, mais les met progressivement en évidence. Ce faisant, il adopte une attitude philosophique résolument réaliste.

6. Grothendieck a souvent recours au gras dans Récoltes et semailles. 
La structure d'une chose n'est nullement une chose que nous puissions «inventer». Nous pouvons seulement la mettre à jour patiemment, humblement en faire connaissance, la "découvrir». S'il y a inventivité dans ce travail, et s'il nous arrive de faire œuvre de forgeron ou d'infatigable bâtisseur, ce n'est nullement pour «façonner», ou pour «bâtir», des «structures". Celles-ci ne nous ont nullement attendues pour être, et pour être exactement ce qu'elles sont! Mais c'est pour exprimer, le plus fidèlement que nous le pouvons, ces choses que nous sommes en train de découvrir et de sonder, et cette structure réticente à se livrer, que nous essayons à tâtons, et par un langage encore balbutiant peut-être, à cerner. [Grothendieck 1985, p. P27]

Dans cette optique, deux tâches fondamentales organisent le travail mathématique. La première est le développement du réseau de concepts Grothendieck parle du langage - par l'entremise duquel la réalité mathématique est appréhendée. La seconde est l'organisation de ces concepts en théories de façon à saisir la structure intime des mathématiques. La suite de la citation précédente décrit brièvement ces deux tâches :

Ainsi sommes-nous amenés à constamment «inventer» le langage apte à exprimer de plus en plus finement la structure intime de la chose mathématique, et à «construire» à l'aide de ce langage, au fur et à mesure et de toutes pièces, les «théories" qui sont censées rendre compte de ce qui a été appréhendé et vu. Il y a là un mouvement de va-et-vient continuel, ininterrompu, entre l'appréhension des choses, et l'expression de ce qui est appréhendé, par un langage qui s'affine et se re-crée au fil du travail, sous la constante pression du besoin immédiat. [Grothendieck 1985, p. P27]

À la lumière de cette conception du travail mathématique, le point de vue du nombre est utilisé pour saisir la structure du discret alors que le point de vue de la grandeur permet de saisir la structure du continu. Il en résulte que l'arithmétique et l'analyse chapeautent respectivement l'étude du discret et celle du continu.

On peut dire que «le nombre» est apte à saisir la structure des agrégats "discontinus", ou "discrets": les systèmes, souvent finis, formés d'éléments ou "objets» pour ainsi dire isolés les uns par rapport aux autres, sans quelque principe de "passage continu " de l'un à l'autre. "La grandeur» par contre est la qualité par excellence, susceptible de "variation continue"; par là, elle est apte à saisir les structures et phénomènes continus: les mouvements, espaces, "variétés » en tous genres, champs de force etc. Ainsi, l'arithmétique apparaît (grosso-modo $[\mathrm{sic}]$ ) comme la science des structures discrètes, et l'analyse, comme la science des structures continues, [sic] [Grothendieck 1985, p. P28]

Pour sa part, l'aspect géométrique ne peut être associé exclusivement à un type de structures au même titre que les aspects arithmétique et analytique. La géométrie recoupe à la fois l'étude des structures discrètes et des structures continues.

Grothendieck rappelle que, traditionnellement, l'étude des figures géométriques pouvait se faire de deux points de vue distincts. Premièrement, la 
topologie combinatoire telle qu'elle se développa dans la foulée de la formule d'Euler s'attardait aux propriétés discrètes des figures. Deuxièmement, la géométrie - analytique ou synthétique, distinction que le Programme d'Erlangen de Klein rendit caduque - examinait les propriétés continues de ces mêmes figures et se basait notamment sur l'idée de grandeur exprimée en termes de distance entre deux points. Ainsi, la géométrie étudiait à la fois le discret et le continu, mais de manière indépendante.

De plus, le développement de la géométrie algébrique abstraite au $\mathrm{xx}^{\mathrm{e}}$ siècle inaugura un renouvellement de l'aspect de la forme en imposant un unique point de vue qui participe simultanément à l'étude des structures discrètes et continues. Pour Grothendieck, l'idée-clé de la géométrie algébrique est la transposition de la géométrie classique, construite sur le corps des réels ou des complexes, à des corps discrets. En effet, étant donné un nombre premier $p$, une théorie de caractéristique $p$ peut être construite. La géométrie algébrique considère ainsi des théories calquées sur la géométrie classique, mais dans un contexte discret. Dans le cadre de la géométrie algébrique abstraite, les structures continues et discrètes ne peuvent être considérées indépendamment les unes des autres.

La mathématique unifiée de Grothendieck s'inscrit dans la continuité de ce renouvellement.

\subsection{Les conjectures de Weil ou le nécessaire rapprochement du discret et du continu}

La géométrie algébrique abstraite fut à l'origine l'œuvre du mathématicien français André Weil. Dans une note aux Comptes rendus de l'Académie des sciences de 1940, Weil annonça être en mesure de résoudre l'hypothèse de Riemann pour les courbes de genre arbitraire. La pierre de touche de la solution de Weil fut la perspective inédite - géométrique il va sans dire sur la fonction zêta que lui donna l'élaboration de la géométrie algébrique abstraite sur des corps finis. [Houzel 1994, p. 401]

Les recherches subséquentes de Weil le conduisirent à formuler dans un article de 1949 quatre conjectures, communément appelées conjectures de Weil. Pour les besoins de la suite, il suffit de savoir que les conjectures portent sur la fonction zêta des variétés algébriques sur des corps finis ${ }^{7}$.

Ayant formulé ce problème, Weil avança une piste de solution: l'existence d'une théorie cohomologique propre aux variétés algébriques sur un corps fini permettrait de les démontrer. Plus précisément, l'idée est de construire une théorie cohomologique qui satisferait certaines conditions topologico-fonctorielles ${ }^{8}$ de façon à avoir les mêmes propriétés que les théories cohomologiques des variétés algébriques sur le corps des complexes $\mathbb{C}$.

7. Pour plus de détails sur les conjectures elles-mêmes, voir Houzel [1994, p. 409]

8. Voir Houzel [1994 , p. 410] pour les conditions. 
Or, si Weil savait que l'existence d'une telle théorie fournirait une solution, la construction de cette théorie n'en représentait pas moins un défi considérable. Mathématiquement, au-delà de la très grande généralité des résultats recherchés, la difficulté inhérente aux conjectures tenait à ce qu'elles suggéraient l'application d'invariants topologiques - les groupes de cohomologie - à des variétés algébriques. Autrement dit, des méthodes relevant de l'étude du continu devraient être appliquées à des espaces discrets.

Selon Grothendieck, les conjectures de Weil exigeaient la construction d'un pont entre les structures discrètes et les structures continues. En postulant une réconciliation du continu et du discret, elles mettaient donc en lumière la nécessité d'une refonte de la géométrie algébrique qui déboucherait sur une nouvelle géométrie façonnée sur mesure pour satisfaire cette exigence inédite.

Les conjectures de Weil jouèrent un rôle fondamental dans l'élaboration de la nouvelle géométrie envisagée par Grothendieck. Premièrement, l'essentiel des travaux de Grothendieck entre 1958 et 1970 forment le cadre technique qui rendit possible la résolution des conjectures en 1973 par Deligne. Deuxièmement et plus important encore, les conjectures de Weil servirent de guide à l'élaboration de cette nouvelle géométrie. Elles représentaient un test évitant le dérapage ou l'effondrement de la construction globale. En effet, la réconciliation du discret et du continu sous-jacente aux conjectures de Weil laissait entrevoir un territoire mathématique inexploré que Grothendieck s'appliquerait à cartographier. Les conjectures constituaient son unique point de repère dans cette tâche. Ainsi, tant que la théorie qu'il s'affairait à construire permettait d'avancer dans la démonstration des conjectures, celle-ci demeurait susceptible de réaliser la vision unificatrice. Comme l'écrit Cartier, "Mais pour Grothendieck, les conjectures de Weil ne sont pas tant intéressantes en elles-mêmes que comme test de la solidité de ses conceptions générales. » [2000, p. 17]

Cette refonte de la géométrie algébrique abstraite débouchera ce que Grothendieck appelle dans Récoltes et semailles la géométrie arithmétique.

\subsection{L'unité dans le contexte de la géométrie arithmétique}

La vision unificatrice des mathématiques qui anime les recherches de Grothendieck s'incarne dans la géométrie arithmétique. Dans cette optique, la motivation derrière l'élaboration de cette dernière apparaît comme l'accomplissement de cette synthèse du discret et du continu.

On peut considérer que la géométrie nouvelle est avant toute autre chose, une synthèse entre ces deux mondes, jusque là [sic] mitoyens et étroitement solidaires, mais pourtant séparés: le monde "arithmétique", dans lequel vivent les (soi-disants) «espaces» sans principe de continuité, et le monde de la grandeur continue, où vivent les «espaces» au sens propre du terme, accessibles aux moyens de l'analyste et (pour cette raison même) acceptés par lui comme dignes 
de gîter dans la cité mathématique. Dans la vision nouvelle, ces deux mondes jadis séparés, n'en forment plus qu'un seul. [Grothendieck 1985, p. P30]

À cette fin, Grothendieck doit cependant se munir d'une nouveau concept d'espace, mais plus précisément de l'espace dans sa compréhension topologique. Le concept d'espace topologique traditionnel, c'est-à-dire celui hérité de Hausdorff, est effectivement inadéquat compte tenu des exigences que devrait satisfaire la théorie cohomologique postulée par les conjectures de Weil.

Ce problème est d'abord technique. Le concept traditionnel d'espace ne possède pas la souplesse requise pour saisir les invariants topologiques de la géométrie arithmétique, c'est-à-dire les groupes de cohomologie qui rendront compte de la forme des variétés algébriques sur un corps fini.

Or, le problème est également conceptuel puisqu'aucun concept d'espace n'était plus général que celui-là. Encore dans les années 1950, une topologie est considérée comme la structure la plus dépouillée et donc la plus générale dont peut disposer un espace. En effet, ce concept englobe tous les autres concepts d'espace. Pour donner un exemple simple, tout espace métrique est un espace topologique, mais l'inverse n'est pas vrai. Conséquemment, même la structure spatiale la plus souple à la disposition des mathématiciens ne l'était pas suffisamment pour le problème que soulevaient les conjectures.

La pierre angulaire de la nouvelle géométrie ne pouvait donc être qu'un concept d'espace permettant de transcender la généralité maximale du concept d'espace topologique traditionnel. En définissant le concept de topos, Grothendieck parvint à se donner un concept d'espace possédant la souplesse requise par les conjectures de Weil.

C'était l'apparition d'un tel «principe nouveau » décidément, et rien de moins, qui pouvait faire se consommer ces «épousailles du nombre et de la grandeur» ou de la "géométrie du discontinu » avec celle du "continu ", dont un premier pressentiment se dégageait des conjectures de Weil. [Grothendieck 1985, p. P34]

Le concept de topos fournira à Grothendieck la généralisation recherchée.

L'idée de la généralisation consiste à remplacer le treillis des ouverts $\mathcal{O}(X)$ qui, par définition, détermine la structure d'un espace topologique au sens traditionnel. Pour ce, Grothendieck a recours à la notion de faisceau. Intuitivement, un faisceau est un outil permettant de décrire une structure mathématique définie localement sur un espace $X$ par un processus de restrictions et de collage. Par exemple, soient un espace topologique $X$ et une fonction $f: U \rightarrow \mathbb{R}$ où $U \in \mathcal{O}(X)$. On considère les restrictions $\left.f\right|_{V}$ sur les sousensembles ouverts $V \subset U$. En choisissant correctement certains sous-ensembles 
ouverts $V_{i}$, il est possible de retrouver la fonction $f$ en collant les restrictions $\left.f\right|_{v i}{ }^{9}$.

Tout d'abord, Grothendieck considère la totalité des faisceaux $F: \mathcal{O}(X)^{\mathrm{op}} \rightarrow$ Ens sur un espace topologique $X$. Ces faisceaux forment une catégorie: la catégorie des faisceaux sur l'espace $X$, dénotée $\operatorname{Sh}(X)$. Cette catégorie est fondamentale: elle permet de retrouver la structure topologique de l'espace — c'est-à-dire le treillis des ouverts $\mathcal{O}(X)$ — et du coup les propriétés essentielles de l'espace. Ceci signifie que la structure topologique de l'espace $X$ est en fait déterminée par la catégorie des faisceaux. Autrement dit, il est possible de faire abstraction de l'espace de départ pour ne se préoccuper que de la catégorie $\operatorname{Sh}(X)^{10}$.

La construction de la catégorie des faisceaux opère donc un passage d'une situation topologique, c'est-à-dire qui relève du continu, vers une situation catégorico-algébrique et donc foncièrement discrète. L'intérêt de ce passage tient à ce que la structure catégorico-spatiale est beaucoup plus souple que la structure topologique. En fait, elle possède la souplesse recherchée par Grothendieck pour transcender la désormais apparente généralité maximale du concept d'espace topologique traditionnel.

Parallèlement, Grothendieck définit une notion de topologie ancrée dans le langage de la théorie des catégories. Elle a l'avantage d'être plus générale et plus souple que la notion ensembliste traditionnelle. Cette topologie «nouveau genre» fait appel à la notion de crible.

Définition. Soient $\mathcal{C}$ une petite catégorie et $C$ un objet de $\mathcal{C}$. Un crible $S$ sur $C$ est une famille de morphismes dans $\mathcal{C}$, chacun ayant $C$ pour codomaine, telle que si $f \in S$, alors $f \circ g \in S$ pour tout morphisme $g$ dont la composition avec $f$ est définie.

Plus simplement, un crible est une famille de morphismes fermée sous la composition à droite. De plus, si $S$ est un crible sur $C$ et $h: D \rightarrow C$ est un morphisme, alors

$$
h^{*}(S)=\{g \mid \operatorname{cod}(g)=D \text { et } h \circ g \in S\}
$$

est un crible sur $D$.

Une des particularités des topologies de Grothendieck, puisqu'il s'agit du nom que porte cette nouvelle notion, est d'être définies sur une catégorie et non sur un ensemble. Grosso modo, une topologie de Grothendieck sur un catégorie $\mathcal{C}$ est une fonction qui associe à tout objet $C$ de $\mathcal{C}$ une collection de cribles sur $C$ et qui respecte certaines propriétés. La définition rigoureuse s'énonce comme suit:

9. Pour une présentation technique de la notion de faisceau, voir Mac Lane et Moerdijk [1992].

10. Pour le détail technique, voir Mac Lane et Moerdijk [1992]. 
Définition. Une topologie de Grothendieck sur une catégorie $\mathcal{C}$ est une fonction $J$ qui assigne à tout objet $C$ de $\mathcal{C}$ une collection $J(C)$ de cribles sur $C$ tel que

1) le crible maximal $t_{C}=\{f \mid \operatorname{cod}(f)=C\}$ est dans $J(C)$;

2) si $S \in J(C)$, alors $h^{*}(S) \in J(D)$ pour tout morphisme $h: D \rightarrow C$;

3) si $S \in J(C)$ et $R$ est un crible sur $C$ tel que, pour tout $h: D \rightarrow C, b^{*}(R) \in J(D)$, alors $R \in J(C)$.

La notion de topologie de Grothendieck permet à son tour de définir un site.

Définition. Un site est une paire $(\mathcal{C}, J)$ où $\mathcal{C}$ une petite catégorie et $J$ une topologie de Grothendieck sur $\mathcal{C}$.

La notion de site rend possible l'étude de la catégorie $\mathcal{C}$ d'un point de vue local. En ce sens, elle remplit un rôle similaire à celui des voisinages pour les espaces topologiques. Il est d'ailleurs facile de démontrer qu'un espace topologique au sens traditionnel est en fait un cas particulier de site ${ }^{11}$. La structure de topologie de Grothendieck généralise donc bel et bien la structure du treillis des ouverts.

Finalement, la construction de la catégorie des faisceaux $\operatorname{Sh}(X)$ peut être transposée de manière à construire la catégorie des faisceaux $\operatorname{Sh}(\mathcal{C}, J)$ sur un site $(\mathcal{C}, J)$. Ceci donne lieu à la notion de topos:

Définition. Un topos $\mathcal{E}$ est une catégorie de faisceaux qui est équivalente à la catégorie des faisceaux $\operatorname{Sh}(\mathcal{C}, J)$ sur un site $(\mathcal{C}, J)$.

Ainsi, le concept de topos fournit la généralisation recherchée du concept d'espace topologique. Comme l'écrit Grothendieck dans Récoltes et semailles:

(...) un "espace nouveau style [", sic] (ou topos), généralisant les espaces topologiques traditionnels, sera décrit tout simplement comme une "catégorie » qui, sans provenir forcément d'un espace ordinaire, possède néanmoins toutes ces bonnes propriétés (explicitement désignées une fois pour toutes, bien sûr) d'une telle «catégorie de faisceaux». [1985, p. P39]

Le topos est la pierre angulaire de la vision unificatrice qui sous-tend l'élaboration de la géométrie arithmétique. Les travaux de Grothendieck établiront que, étant donné une variété algébrique de caractéristique $p$, le topos permet de construire la théorie cohomologique postulée par Weil qu'il nommera cohomologie étale $l$-adique.

En conséquence, dans la géométrie arithmétique telle que développée par Grothendieck, l'unité des mathématiques s'organise autour du concept de topos.

11. Voir Mac Lane etMoerdijk [1992, p. 111] pour une démonstration. 
[L'idée de topos] englobe, dans une intuition topologique commune, aussi bien les traditionnels espaces (topologiques), incarnant le monde de la grandeur continue, que les (soi-disant) «espaces» (ou "variétés») des géomètres algébristes abstraits impénitents, ainsi que d'innombrables autres types de structures, qui jusque là [sic] avaient semblé rivées irrémédiablement au «monde arithmétique» des agrégats "discontinus» ou «discrets». [Grothendieck 1985, p. P37]

Plus généralement, ceci signifie que l'outil topossique, en permettant d'appliquer des invariants topologiques à une variété algébrique sur un corps fini, fait de la géométrie un pont entre les aspects arithmétique et analytique des mathématiques, c'est-à-dire entre le discret et le continu.

C'est le thème du topos (...) qui est ce «lit », ou cette "rivière profonde ", où viennent s'épouser la géométrie et l'algèbre, la topologie et l'arithmétique, la logique mathématique et la théorie des catégories, le monde du continu et celui des structures «discontinues» ou «discrètes ». [Grothendieck 1985, p. P43]

En conclusion, l' «Essai sur l'unité des sciences mathématiques dans leur développement actuel» de Lautman adopte un point de vue philosophique original sur l'unité des mathématiques. Celui-ci peut effectivement être qualifié de métaphysique puisque l'unité est le résultat des schémas logiques à l'œuvre en mathématiques.

Paradoxalement, le thème d'une unité métaphysique n'est introduit qu'à la toute fin de la thèse complémentaire. Il demeure par conséquent sous-développé et difficile à interpréter, et ce encore plus si celle-ci est étudiée indépendamment de l' "Essai sur les notions de structure et d'existence en mathématique ». L'introduction et les quatre chapitres annoncent plutôt une unité méthodologique qui se baserait ultimement sur une réconciliation du continu et du discret.

Dans une perspective mathématique, l'unité induite par le concept de topos dans le cadre des recherches de Grothendieck s'avère beaucoup plus satisfaisante. Ceci ne doit toutefois pas nécessairement être compris comme un rejet définitif du traitement de Lautman de la dualité du continu et du discret - les solutions de Lautman et Grothendieck répondent après tout à des préoccupations fort différentes. Toutefois, malgré sa difficulté technique, la clarté de l'approche de Grothendieck l'avantage.

Finalement, à la lumière de la vaste infiltration du concept de topos dans plusieurs champs des mathématiques depuis les années 1960, il serait certainement intéressant d'approfondir la nouvelle portée qu'acquérait une conception de l'unité des mathématiques basée sur les topos. 


\section{Bibliographie}

Bernays, Paul (1940). "Review of “Essai sur l'unité des sciences mathématiques dans leur développement" ", The Journal of Symbolic Logic, 5, 1, p. 22.

Cartier, Pierre (2000). "Grothendieck et les motifs", Prépublications de l'Institut des Hautes Études Scientifiques, coll. Notes sur l'histoire et la philosophie des mathématiques, ${ }^{\circ}$ IV, p. 2-34.

Grothendieck, Alexandre (1985). Récoltes et semailles, Montpellier, Université des Sciences et Technologies du Languedoc.

Houzel, Christian (1994). «La Préhistoire des conjectures de Weil» dans Pier, JeanPaul, Development of Mathematics 1900-1950, Basel, Birkhäuser, p. 385414.

Lautman, Albert (1977a). «Essai sur les notions de structure et d'existence en mathématique", dans Essai sur l'unité des mathématiques et divers écrits, Paris, Union générale d'éditions, coll. 10/18, p. 23-154.

Lautman, Albert (1977b). «Essai sur l'unité des mathématiques dans leur développement actuel ", dans Essai sur l'unité des mathématiques et divers écrits, Paris, Union générale d'éditions, coll. 10/18, p. 155-202.

Mac Lane, Saunders et Ieke Moerdijk (1992). Sheaves in Geometry and Logic: A First Introduction to Topos Theory, New York, Springer, coll. Universitext. 